\title{
Two-current correlations in the pion in the Nambu and Jona-Lasinio model
}

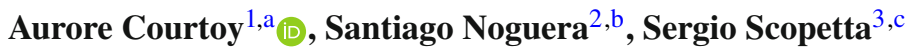 \\ ${ }^{1}$ Instituto de Física, Universidad Nacional Autónoma de México, Apartado Postal 20-364, 01000 Ciudad de México, Mexico \\ ${ }^{2}$ Departamento de Física Teórica and IFIC, Centro Mixto Universidad de Valencia-CSIC, 46100 Burjassot, Valencia, Spain \\ ${ }^{3}$ Dipartimento di Fisica e Geologia, Università degli Studi di Perugia, and Istituto Nazionale di Fisica Nucleare Sezione di Perugia, Via A. Pascoli, \\ 06123 Perugia, Italy
}

Received: 17 June 2020 / Accepted: 12 September 2020 / Published online: 1 October 2020

(c) The Author(s) 2020

\begin{abstract}
We present an analysis of two-current correlations for the pion in the Nambu-Jona-Lasinio model, with Pauli-Villars regularization. We provide explicit expressions in momentum space for two-current correlations corresponding to the zeroth component of the vector Dirac bilinear in the quark vertices, which has been evaluated on the lattice, thinking to applications in a high energy framework, as a step towards the calculation of pion double parton distributions. The numerical results show a remarkable qualitative agreement with recent lattice data. The factorization approximation into one-body currents is discussed based on previous evaluation of the relevant low energy matrix elements in the Nambu-Jona-Lasinio model, confirming the lattice result.
\end{abstract}

\section{Introduction}

Due to the high partonic densities reached, processes with more than two partons from the two colliding protons participating in the actual scattering - the so called multiple parton interactions - are likely to happen at the LHC [1]. Double parton scattering (DPS), the simplest form of multiple parton interactions, involves two simultaneous hard collisions. It has been indeed observed at the LHC (see, e.g., Ref. [2]). The DPS cross section is expressed in terms of double parton distribution functions (dPDFs) [3,4]. The latter are related to the number density of two partons located at a given transverse separation in coordinate space with given longitudinal momentum fractions. The information encoded in these distributions therefore complements that accessed through electromagnetic probes in terms of generalized parton distribu-

\footnotetext{
a e-mail: aurore@ fisica.unam.mx (corresponding author)

be-mail: Santiago.Noguera@uv.es

c e-mail: Sergio.Scopetta@pg.infn.it
}

tions (GPDs) [5,6], one of the flagships of the future Electron Ion Collider (EIC) [7]. If measured, dPDFs would therefore represent a novel tool to study the three-dimensional hadron structure [8-12]. Indeed, they are sensitive to two-parton correlations not accessible via one body distributions, e.g. Parton Distribution Functions (PDFs) and GPDs -see Ref. $[1,13]$ for a recent report. Since they are non perturbative objects, dPDFs have been evaluated within models of the hadron structure - estimates exist at low momentum scales for the proton dPDFs [14-20]. In order to match theoretical predictions with future experimental analyses, the results of these calculations are then evolved using the proper QCD evolution equations to reach the high momentum scale of the data. Developments on the evolution properties of dPDFs can be found in, e.g., [21].

Recently, the double PDFs of the pion have also attracted considerable attention. A first estimate has been performed using light cone wave functions obtained within the AdS/QCD correspondence $[22,23]$; then a calculation within a NambuJona-Lasinio (NJL) framework was presented in Ref. [24]. The latter represents the first evaluation of pion dPDFs in a field theoretical approach, which allowed a systematical study of the different contributions to the pion observables. This exercise has been effective to examine the validity of commonly used approximations for which dPDFs could be expressed in terms of GPDs - and were found to be violated, especially at low momentum scale and in the valence region. These results have been recently confirmed in a similar NJL framework by an independent calculation [25].

Studies for the pion are especially important in view of possible lattice calculations. This is pertinent in particular when a corresponding evaluation for nucleon observables on the lattice appear much more involved. However, the communities studying parton distributions from global QCD analyses and lattice QCD calculations are collaborating towards 
a synergy to further improve our knowledge of PDFs [26], thanks to the access -on the lattice- to moments of PDFs as well as to the formalism of quasi-PDFs and its matching procedure to lightfront PDFs, e.g. [27]. This effort is expanding to all collinear PDFs, GPDs and Transverse momentum dependent parton distributions (TMDs) of the nucleons [28]. Since similar considerations are premature for multi parton PDFs, it seems opportune to consider the two-current correlations in the pion - quantities that could be related to dPDFs through moments - with current operators evaluated at different space points but at equal time, from a model point of view. Such correlations have been calculated on the lattice, e.g., in Ref. [29] and more recently in Refs. [30,31].

The novel possibility to compare results with lattice data reinforces the theoretical relevance of model calculations of two-current correlations in the pion.

In this paper we analyze the correlations of two vector currents in the NJL model, showing in particular their time-time component, and attempting for this quantity a direct comparison with lattice data. We remark that the lattice calculation has been performed as an intermediate step towards the evaluation of dPDFs and, therefore, only contributions relevant in a high energy scenario have been taken into account. To have a consistent comparison, in our NJL evaluation we have neglected possible contributions to the two current correlations which can be important at low energies but are suppressed in DIS and related phenomena. We follow therefore the lines successfully developed in Refs. [32-42].

The paper is structured as follows. In Sect. 2 we define two-current correlations in the pion and their isospin decomposition, while in Sect. 3 we describe the NJL evaluation scheme and we give the explicit expressions of the results of the calculation. In Sect. 4 we show a comparison of our numerical results with lattice data. Conclusions are collected in Sect. 5.

\section{Definitions and isospin decomposition}

Experimental measurements of pion dPDFs appear rather challenging at the moment. Nevertheless, from a complementary viewpoint, lattice evaluations of matrix elements that can be connected to the Mellin moments of double parton distributions are possible [4]. A relevant step towards this goal has been done in Ref. [31], where lattice data of correlation functions of two local currents in a pion,

$\left\langle\pi^{k}(P)\left|\mathcal{O}_{i}^{q_{1} q_{2}}(y) \mathcal{O}_{j}^{q_{3} q_{4}}(0)\right| \pi^{k^{\prime}}(P)\right\rangle$,

have been presented. In the expression above, the pion charges $-k, k^{\prime}=+,-, 0-$ may be different in the bra and ket state, while the four-momentum $P$ is the same in both, and the two currents are applied at the same time but at different spatial positions, i.e., one has $y^{\mu}=(0, \mathbf{y})$. It differs from the dPDFs which are defined from a similar product of two one body operators taken at a distance $y^{\prime \mu}=\left(y^{\prime+}=0, y^{-}, \mathbf{y}_{\perp}^{\prime}\right)$. Hence, the currents of interest here are

$\mathcal{O}_{n}^{q q^{\prime}}(y)=\bar{q}(y) \Gamma_{n} q^{\prime}(y)$,

where $q$ and $q^{\prime}$ are $u$ or $d$ quark fields and $\Gamma_{n}$ are the Dirac bilinears $1, i \gamma_{5}, \gamma^{\mu}, \gamma^{\mu} \gamma_{5}$ for $n=S, P, V^{\mu}, A^{\mu}$, respectively. In the present work we will only consider the time component of the vector current, i.e.

$\mathcal{O}_{V^{0}}^{q q^{\prime}}(y)=\bar{q}(y) \gamma^{0} q^{\prime}(y)$,

and we will compare our model results with Lattice data in the case of two equal currents of this kind.

As shown in Ref. [31], the matrix elements (1) are not all independent due to constraints from isospin symmetry and discrete symmetries. We follow the treatment of Ref. [31] defining the isosinglet current:

$\mathcal{O}_{n}^{\mathrm{s}}=\mathcal{O}_{n}^{u u}+\mathcal{O}_{n}^{d d}$,

as well as the isotriplet currents

$\mathcal{O}_{n}^{a}=\bar{Q} \tau^{a} \Gamma_{n} Q, \quad a=1,2,3$

where $\tau^{a}$ are the Pauli matrices and $Q=(u, d)$ is the isodoublet of quark fields. As a consequence, the following isospin decomposition is obtained

$$
\begin{aligned}
\left\langle\pi^{j}(P)\left|\mathcal{O}_{n}^{\mathrm{s}}(y) \mathcal{O}_{n^{\prime}}^{\mathrm{s}}(0)\right| \pi^{i}(P)\right\rangle= & \delta^{i j} F_{0}(y) \\
\left\langle\pi^{j}(P)\left|\mathcal{O}_{n}^{a}(y) \mathcal{O}_{n^{\prime}}^{b}(0)\right| \pi^{i}(P)\right\rangle= & \delta^{a b} \delta^{i j} F_{1}(y) \\
& +\left(\delta^{a i} \delta^{b j}+\delta^{a j} \delta^{b i}\right) F_{2}(y) \\
& +\left(\delta^{a i} \delta^{b j}-\delta^{a j} \delta^{b i}\right) i F_{3}(y), \\
\left\langle\pi^{j}(P)\left|\mathcal{O}_{n}^{b}(y) \mathcal{O}_{n^{\prime}}^{\mathrm{s}}(0)\right| \pi^{i}(P)\right\rangle= & i \epsilon^{b i j} G_{1}(y) \\
\left\langle\pi^{j}(P)\left|\mathcal{O}_{n}^{\mathrm{s}}(y) \mathcal{O}_{n^{\prime}}^{b}(0)\right| \pi^{i}(P)\right\rangle= & i \epsilon^{b i j} G_{2}(y)
\end{aligned}
$$

Here the real functions $F_{\ell}$ and $G_{\ell}$ - quantities evaluated in what follows - depend on the pion momentum $P$ and on the current indices $n, n^{\prime}$ [31].

\section{Two-current correlations in the NJL model}

\subsection{Leading order}

We describe now the calculation of the two-current correlations, and in particular of the functions $F_{i}$, with $i=0,1,2$, 
and $G_{l}$, with $l=1,2$, in the NJL framework. This framework is the most realistic model for the pseudoscalar mesons based on a local quantum field theory built only with quarks, see Ref. [43] for a review and Refs. [44-46] for low-energy applications beyond the partonic structure of hadrons. It respects the realization of chiral symmetry and gives a good description of low energy properties. Mesons are described as bound states, in a fully covariant fashion, using the BetheSalpeter amplitude, in a field theoretical framework. In this way, Lorentz covariance is preserved. The NJL model is a non-renormalizable field theory and therefore a regularization procedure has to be implemented. We have performed our calculations in the Pauli-Villars regularization scheme - a well established method. The NJL model, together with its regularization procedure, can be regarded as an effective theory of QCD. Some basic features of the NJL model and details on the regularization scheme are reported in Appendix A. Model calculations of meson partonic structure within this approach have a long story of successful predictions [32-42].

Collinear parton distributions evaluated within a low energy model can be associated to a RGE scale denoted by $Q_{0}$. The determination of that hadronic scale reflects the degrees of freedom of the model - here the NJL model contains only valence quarks $[47,48]$. The identification of such a low lying scale enables predictions from the model to measured quantities, through perturbative QCD evolution.

Let us describe the main steps of our calculation. We define, following Eq. (6),

$G_{\alpha \beta}^{i j}(\mathbf{y})=\left\langle\pi^{j}(P)\left|\mathcal{O}_{n}^{\beta}(y) \mathcal{O}_{n^{\prime}}^{\alpha}(0)\right| \pi^{i}(P)\right\rangle$,

with $\alpha, \beta=\mathrm{s}, 1,2,3$. In order to proceed to the calculation in the NJL model, we perform the Fourier transform to momentum space

$$
\begin{aligned}
& G_{\alpha \beta}^{i j}(\mathbf{q}) \\
& =\int d^{3} y e^{-i \mathbf{q} \cdot \mathbf{y}} G_{\alpha \beta}^{i j}(\mathbf{y}) \\
& =\int d^{3} y e^{-i \mathbf{q} \cdot \mathbf{y}}\left\langle\pi^{j}(P)\left|\mathcal{O}_{n}^{\beta}(y) \mathcal{O}_{n^{\prime}}^{\alpha}(0)\right|_{y^{0}=0} \mid \pi^{i}(P)\right\rangle .
\end{aligned}
$$

We use in this expression the following definition of the state for the pion $i$

$$
\begin{aligned}
\left|\pi^{i}(P)\right\rangle= & \int d^{4} y_{1} d^{4} y_{2} \frac{d^{4} k}{(2 \pi)^{4}} e^{-i \frac{1}{2} P \cdot\left(y_{1}+y_{2}\right)} e^{-i k \cdot\left(y_{1}-y_{2}\right)} \\
& \times \bar{q}\left(y_{1}\right) \phi_{\pi^{i}}(k, P) q\left(y_{2}\right)|0\rangle
\end{aligned}
$$

with $\phi_{\pi^{i}}$ the quark-pion vertex function for a $\pi^{i}$. In the NJL model the amplitude $\phi_{\pi^{i}}(k, P)$ is independent on the relative and total quark-antiquark momenta, $k$ and $P$, respectively, and we have

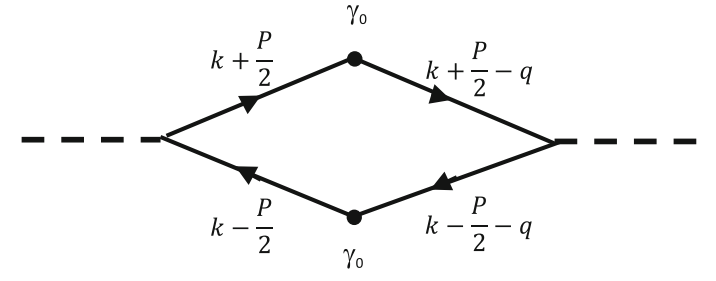

a

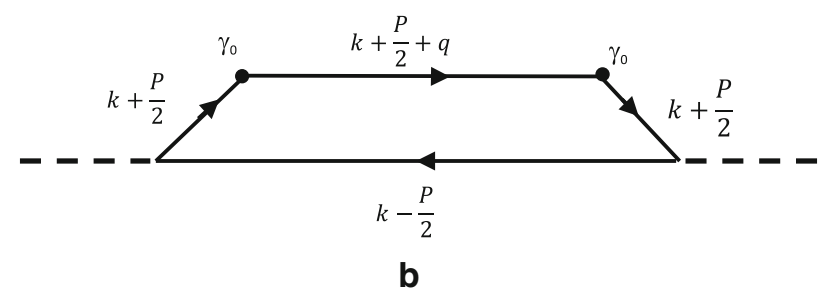

Fig. 1 Examples of the two types of diagrams contributing to the leading order of the time component of the two local vector current correlations: rhombus (a) and trapezoid (b)

$\phi_{\pi^{i}}(k, P)=i g_{\pi q q} i \gamma_{5} \tau^{i}$,

where $g_{\pi q q}$ is the quark-pion coupling constant and $\tau^{i}$ is the isospin matrix associated to the corresponding pion $\pi^{i}$.

Inserting Eq. (9) in the definition (8), we obtain contributions associated to different Feynman diagrams. The fact that $G_{\alpha \beta}^{i j}(\mathbf{y})$ is defined at $y^{0}=0$ implies that

$G_{\alpha \beta}^{i j}(\mathbf{q})=\int \frac{d q^{0}}{2 \pi} \mathcal{T}$,

where $\mathcal{T}$ is the Feynman amplitude corresponding to the diagram.

The two contributions to be considered at leading order are shown in Fig. 1. In the following, we will call them rhombus and trapezoid diagrams, respectively. Therefore one has

$G_{\alpha \beta}^{(0) i j}(\mathbf{q})=G_{\alpha \beta}^{(r) i j}(\mathbf{q})+G_{\alpha \beta}^{(t) i j}(\mathbf{q})$,

where $G_{\alpha \beta}^{(r) i j}(\mathbf{q})$ and $G_{\alpha \beta}^{(t) i j}(\mathbf{q})$ stand for contributions from the rhombus and trapezoid diagrams, respectively, which read

$$
\begin{aligned}
G_{\alpha \beta}^{(r) i j}(\mathbf{q})= & \int \frac{d^{4} k}{(2 \pi)^{4}} \frac{d q^{0}}{2 \pi}(-) \\
& \times\left\{\operatorname { T r } \left[\bar{\phi}_{\pi^{j}} i S_{F}\left(\frac{P}{2}+k+q\right) \tau^{\alpha} \Gamma_{n^{\prime}} i S_{F}\right.\right. \\
& \times\left(k+\frac{P}{2}\right) \phi_{\pi^{i}} i S_{F}\left(k-\frac{P}{2}\right) \tau^{\beta} \Gamma_{n} i S_{F} \\
& \left.\times\left(k+q-\frac{P}{2}\right)\right] \\
& \left.+\left(q^{\mu} \leftrightarrow-q^{\mu},\left(\alpha, n^{\prime}\right) \leftrightarrow(\beta, n)\right)\right\}, \\
G_{\alpha \beta}^{(t) i j}(\mathbf{q})= & \int \frac{d^{4} k}{(2 \pi)^{4}} \frac{d q^{0}}{2 \pi}(-)
\end{aligned}
$$




$$
\begin{aligned}
& \times\left\{\operatorname { T r } \left[\bar{\phi}_{\pi^{j}} i S_{F}\left(\frac{P}{2}+k\right) \tau^{\beta} \Gamma_{n} i S_{F}\right.\right. \\
& \times\left(k+\frac{P}{2}+q\right) \tau^{\alpha} \Gamma_{n^{\prime}} i S_{F}\left(k+\frac{P}{2}\right) \phi_{\pi^{i}} i S_{F} \\
& \left.\times\left(k-\frac{P}{2}\right)\right] \\
& +\operatorname{Tr}\left[\bar{\phi}_{\pi^{j}} i S_{F}\left(\frac{P}{2}+k\right) \phi_{\pi^{i}} i S_{F}\left(k-\frac{P}{2}\right) \tau^{\beta} \Gamma_{n} i S_{F}\right. \\
& \left.\times\left(k-\frac{P}{2}+q\right) \tau^{\alpha} \Gamma_{n^{\prime}} i S_{F}\left(k-\frac{P}{2}\right)\right] \\
& \left.+\left(q^{\mu} \leftrightarrow-q^{\mu},\left(\alpha, m^{\prime}\right) \leftrightarrow(\beta, m)\right)\right\} .
\end{aligned}
$$

Here $\tau^{\alpha}=\left(\tau^{\mathrm{s}}=1, \tau\right)$, with $\tau$ the standard Pauli matrices.

According to their isospin structure, which is determined by $\phi_{i}$ and $\tau^{\alpha}$ terms, the two expressions above yield four types of contributions once the isospin traces are evaluated - a straightforward lengthy procedure. For the rhombus contribution, they read

$$
\begin{aligned}
G_{s s}^{(r) i j}(\mathbf{q})= & \delta_{i j} 2 N_{c}\left(i g_{\pi q q}\right)^{2}\left[I_{\Gamma}^{r}(m, \mathbf{q})+I_{\Gamma}^{r}(m,-\mathbf{q})\right. \\
G_{s a}^{(r) i j}(\mathbf{q})= & 2 i \epsilon_{j i a} N_{c}\left(i g_{\pi q q}\right)^{2}\left[I_{\Gamma}^{r}(m, \mathbf{q})-I_{\Gamma}^{r}(m,-\mathbf{q})\right] \\
G_{a s}^{(r) i j}(\mathbf{q})= & 2 i \epsilon_{i j a} N_{c}\left(i g_{\pi q q}\right)^{2}\left[I_{\Gamma}^{r}(m, \mathbf{q})-I_{\Gamma}^{r}(m,-\mathbf{q})\right] \\
G_{a b}^{(r) i j}(\mathbf{q})= & \left(\delta_{j a} \delta_{i b}-\delta_{j i} \delta_{a b}+\delta_{j b} \delta_{i a}\right) 2 N_{c}\left(i g_{\pi q q}\right)^{2} \\
& \times\left[I_{\Gamma}^{r}(m, \mathbf{q})+I_{\Gamma}^{r}(m,-\mathbf{q})\right]
\end{aligned}
$$

with

$$
\begin{aligned}
I_{\Gamma}^{r}(m, \mathbf{q})= & \int \frac{d^{4} k}{(2 \pi)^{4}} \frac{d q_{0}}{2 \pi} \\
& \times \operatorname{tr}\left[\gamma_{5} S_{F}\left(k+q+\frac{P}{2}\right) \Gamma S_{F}\left(k+\frac{P}{2}\right)\right. \\
& \left.\times \gamma_{5} S_{F}\left(k-\frac{P}{2}\right) \Gamma S_{F}\left(k+q-\frac{P}{2}\right)\right] .
\end{aligned}
$$

In the equations above, $m$ is the quark mass and we have assumed that $\Gamma_{n}=\Gamma_{n^{\prime}}=\Gamma$. For the trapezoid contribution, they read

$$
\begin{aligned}
& G_{s s}^{(t) i j}(\mathbf{q}) \\
&= \delta_{i j} 2 N_{c}\left(i g_{\pi q q}\right)^{2} \\
& \quad \times\left[I_{\Gamma}^{t_{1}}(m, \mathbf{q})+I_{\Gamma}^{t_{1}}(m,-\mathbf{q})+I_{\Gamma}^{t_{2}}(m, \mathbf{q})+I_{\Gamma}^{t_{2}}(m,-\mathbf{q})\right], \\
& G_{s a}^{(t) i j}(\mathbf{q}) \\
&=2 i \epsilon_{j i a} N_{c}\left(i g_{\pi q q}\right)^{2} \\
& \quad \times\left[-I_{\Gamma}^{t_{1}}(m, \mathbf{q})+I_{\Gamma}^{t_{1}}(m,-\mathbf{q})+I_{\Gamma}^{t_{2}}(m, \mathbf{q})-I_{\Gamma}^{t_{2}}(m,-\mathbf{q})\right], \\
& G_{a s}^{(t) i j}(\mathbf{q}) \\
&=2 i \epsilon_{j i a} N_{c}\left(i g_{\pi q q}\right)^{2} \\
& \quad \times\left[-I_{\Gamma}^{t_{1}}(m, \mathbf{q})+I_{\Gamma}^{t_{1}}(m,-\mathbf{q})+I_{\Gamma}^{t_{2}}(m, \mathbf{q})-I_{\Gamma}^{t_{2}}(m,-\mathbf{q})\right], \\
& G_{a b}^{(t) i j}(\mathbf{q}) \\
&=2 N_{c}\left(i g_{\pi q q}\right)^{2}\left\{\delta _ { j i } \delta _ { a b } \left[I_{\Gamma}^{t_{1}}(m, \mathbf{q})+I_{\Gamma}^{t_{1}}(m,-\mathbf{q})+I_{\Gamma}^{t_{2}}(m, \mathbf{q})\right.\right. \\
&\left.\quad+I_{\Gamma}^{t_{2}}(m,-\mathbf{q})\right]+\left(\delta_{j b} \delta_{a i}-\delta_{j a} \delta_{b i}\right)
\end{aligned}
$$

$\left.\times\left[I_{\Gamma}^{t_{1}}(m, \mathbf{q})-I_{\Gamma}^{t_{1}}(m,-\mathbf{q})-I_{\Gamma}^{t_{2}}(m, \mathbf{q})+I_{\Gamma}^{t_{2}}(m,-\mathbf{q})\right]\right\}$,

with

$$
\begin{aligned}
I_{\Gamma}^{t_{1}}(m, \mathbf{q})= & \int \frac{d^{4} k}{(2 \pi)^{4}} \frac{d q^{0}}{2 \pi} \\
& \times \operatorname{tr}\left[\gamma_{5} S_{F}\left(k+\frac{P}{2}\right) \Gamma S_{F}\left(k+\frac{P}{2}+q\right)\right. \\
& \left.\times \Gamma S_{F}\left(k+\frac{P}{2}\right) \gamma_{5} S_{F}\left(k-\frac{P}{2}\right)\right],
\end{aligned}
$$

and

$$
\begin{aligned}
I_{\Gamma}^{t_{2}}(m, \mathbf{q})= & \int \frac{d^{4} k}{(2 \pi)^{4}} \frac{d q^{0}}{2 \pi} \\
& \times \operatorname{tr}\left[\gamma_{5} S_{F}\left(k+\frac{P}{2}\right) \gamma_{5} S_{F}\left(k-\frac{P}{2}\right)\right. \\
& \left.\times \Gamma S_{F}\left(k-\frac{P}{2}+q\right) \Gamma S_{F}\left(k-\frac{P}{2}\right)\right] .
\end{aligned}
$$

By comparing Eqs. (15) and (17) with Eq. (6) we obtain the relations,

$$
\begin{aligned}
F_{0}(\mathbf{q}) & =X_{\Gamma}^{r}(\mathbf{q})+X_{\Gamma}^{t}(\mathbf{q}), \\
F_{1}(\mathbf{q}) & =-X_{\Gamma}^{r}(\mathbf{q})+X_{\Gamma}^{t}(\mathbf{q}) \\
F_{2}(\mathbf{q}) & =X_{\Gamma}^{r}(\mathbf{q}) \\
i F_{3}(\mathbf{q}) & =-Z_{\Gamma}^{t}(\mathbf{q}) \\
G_{1}(\mathbf{q}) & =-Y_{\Gamma}^{r}(\mathbf{q})-Y_{\Gamma}^{t}(\mathbf{q}), \\
G_{2}(\mathbf{q}) & =Y_{\Gamma}^{r}(\mathbf{q})-Y_{\Gamma}^{t}(\mathbf{q})
\end{aligned}
$$

with

$$
\begin{aligned}
X_{\Gamma}^{r}(\mathbf{q})= & 2 N_{c}\left(i g_{\pi q q}\right)^{2}\left[I_{\Gamma}^{r}(m, \mathbf{q})+I_{\Gamma}^{r}(m,-\mathbf{q})\right] \\
Y_{\Gamma}^{r}(\mathbf{q})= & 2 N_{c}\left(i g_{\pi q q}\right)^{2}\left[I_{\Gamma}^{r}(m, \mathbf{q})-I_{\Gamma}^{r}(m,-\mathbf{q})\right], \\
X_{\Gamma}^{t}(\mathbf{q})= & 2 N_{c}\left(i g_{\pi q q}\right)^{2}\left[I_{\Gamma}^{t_{1}}(m, \mathbf{q})+I_{\Gamma}^{t_{2}}(m, \mathbf{q})\right. \\
& \left.+I_{\Gamma}^{t_{1}}(m,-\mathbf{q})+I_{\Gamma}^{t_{2}}(m,-\mathbf{q})\right] \\
Y_{\Gamma}^{t}(\mathbf{q})= & 2 N_{c}\left(i g_{\pi q q}\right)^{2}\left[-I_{\Gamma}^{t_{1}}(m, \mathbf{q})+I_{\Gamma}^{t_{2}}(m, \mathbf{q})\right. \\
& \left.+I_{\Gamma}^{t_{1}}(m,-\mathbf{q})-I_{\Gamma}^{t_{2}}(m,-\mathbf{q})\right], \\
Z_{\Gamma}^{t}(\mathbf{q})= & 2 N_{c}\left(i g_{\pi q q}\right)^{2}\left[I_{\Gamma}^{t_{1}}(m, \mathbf{q})-I_{\Gamma}^{t_{2}}(m, \mathbf{q})\right. \\
& \left.-I_{\Gamma}^{t_{1}}(m,-\mathbf{q})+I_{\Gamma}^{t_{2}}(m,-\mathbf{q})\right] .
\end{aligned}
$$

In Ref. [31] it is shown that $i F_{3}(\mathbf{q})$ is different from zero only if $\Gamma_{n} \neq \Gamma_{n^{\prime}}$, and charge conjugation constraints imply that $G_{\ell}(\mathbf{q})=0$ for the cases $\Gamma_{n}=\Gamma_{n^{\prime}}$. We observe that these results are automatically reproduced if $I_{\Gamma}^{r}(m, \mathbf{q})$ and $I_{\Gamma}^{t_{i}}(m, \mathbf{q})$ are functions of $|\mathbf{q}|$ only. We will see later on that this fact actually occurs in our calculation.

The integrals over $q^{0}$ and $k^{0}$ in the expressions $I_{\Gamma}^{r, t_{i}}(\mathbf{q})$ can be easily evaluated using the poles of the propagators. 
For instance, when $\Gamma=V^{0}=\gamma^{0}$ we obtain

$$
\begin{aligned}
I_{V^{0}}^{r}(m, \mathbf{q})= & \int \frac{d^{3} k}{(2 \pi)^{3}} \\
& \times \frac{4\left[E_{|\mathbf{k}|}^{2}\left(4 E_{|\mathbf{k}+\mathbf{q}|}^{2}+m_{\pi}^{2}\right)+m_{\pi}^{2} \mathbf{k} \cdot \mathbf{q}\right]}{E_{|\mathbf{k}|} E_{|\mathbf{k}+\mathbf{q}|}\left(4 E_{|\mathbf{k}|}^{2}-m_{\pi}^{2}\right)\left(4 E_{|\mathbf{k}+\mathbf{q}|}^{2}-m_{\pi}^{2}\right)},
\end{aligned}
$$

where $E_{v}=\sqrt{v^{2}+m^{2}}$. Now, the angular integration can be performed, arriving to

$$
\begin{aligned}
I_{V^{0}}^{r}(m, q)= & \int \frac{d k}{(2 \pi)^{2}} \frac{k}{q E_{k}} \\
& \times\left\{\left(E_{k+q}-E_{k-q}\right)\left(1+\frac{3}{2} \frac{m_{\pi}^{2}}{4 E_{k}^{2}-m_{\pi}^{2}}\right)\right. \\
& +m_{\pi} \frac{32 E_{k}^{2}-2 E_{k+q}^{2}-2 E_{k-q}^{2}+m_{\pi}^{2}}{8\left(4 E_{k}^{2}-m_{\pi}^{2}\right)} \\
& \left.\times \ln \frac{\left(2 E_{k-q}+m_{\pi}\right)\left(2 E_{k+q}-m_{\pi}\right)}{\left(2 E_{k-q}-m_{\pi}\right)\left(2 E_{k+q}+m_{\pi}\right)}\right\},
\end{aligned}
$$

where $k=|\mathbf{k}|$ and $q=|\mathbf{q}|$. In a similar way we obtain

$$
\begin{aligned}
I_{V^{0}}^{t}(m, q)= & I_{V^{0}}^{t_{1}}(m, q)+I_{V^{0}}^{t_{2}}(m, q) \\
= & \int \frac{d k}{(2 \pi)^{2}}\left(-\frac{k}{q E_{k}}\right)\left(E_{k+q}-E_{k-q}\right) \\
& \times\left\{1-\frac{1}{3} \frac{\left(E_{k+q}-E_{k-q}\right)^{2}}{4 E_{k}^{2}-m_{\pi}^{2}}-\frac{m_{\pi}^{2}}{3\left(4 E_{k}^{2}-m_{\pi}^{2}\right)^{2}}\right. \\
& \left.\times\left[36 E_{k}^{2}-2\left(E_{k+q}-E_{k-q}\right)^{2}-3 m_{\pi}^{2}\right]\right\} .
\end{aligned}
$$

After regularization, the same integrals become

$$
\begin{aligned}
& \bar{I}_{V^{0}}^{r}(q)=\sum_{i=0}^{2} c_{i} I_{V^{0}}^{r}\left(M_{i}, q\right), \\
& \bar{I}_{V^{0}}^{t}(q)=\sum_{i=0}^{2} c_{i} I_{V^{0}}^{t}\left(M_{i}, q\right) .
\end{aligned}
$$

One can see that these integrals do not depend on the direction of $\mathbf{q}$, as anticipated, and therefore Eq. (21) become

$$
\begin{aligned}
& X_{V^{0}}^{r}(q)=4 N_{c}\left(i g_{\pi q q}\right)^{2}\left[\bar{I}_{V^{0}}^{r}(q)\right], \\
& X_{V^{0}}^{t}(q)=4 N_{c}\left(i g_{\pi q q}\right)^{2}\left[\bar{I}_{V^{0}}^{t}(q)\right], \\
& Y_{V^{0}}^{r}(q)=Y_{V^{0}}^{t}(q)=Z_{V^{0}}^{t}(q)=0 .
\end{aligned}
$$

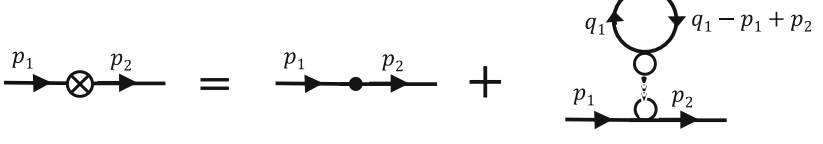

Fig. 2 Dressed local vertex

We conclude this section observing that the above equations define the leading order contributions to the functions (20) in the $\Gamma=\gamma^{0}=V^{0}$ case:

$$
\begin{aligned}
F_{0}(q) & =X_{V^{0}}^{r}(q)+X_{V^{0}}^{t}(q), \\
F_{1}(q) & =-X_{V^{0}}^{r}(q)+X_{V^{0}}^{t}(q), \\
F_{2}(\mathbf{q}) & =X_{V^{0}}^{r}(q), \\
i F_{3}(q) & =0, \\
G_{1}(q) & =0, \\
G_{2}(q) & =0 .
\end{aligned}
$$

\subsection{Dressing the vertex and other contributions}

In addition to the contribution we have just discussed, one has to consider that, in the present NJL scheme, the local vertex can be dressed by the chiral interaction through the diagrams depicted in Fig. 2. This dressing, which could produce corrections to the results obtained in the previous subsection, implies to change the bare vertex $\tau^{\alpha} \Gamma$ as follows, for the isoscalar and isovector vertices respectively

$$
\begin{aligned}
& \Gamma \rightarrow \Gamma+\frac{2 i g}{1-2 g \Pi_{S}\left(q^{2}\right)} \\
& \times\left(-2 N_{c}\right) \int \frac{d^{4} q_{1}}{(2 \pi)^{4}} \operatorname{tr}\left[i S_{F}\left(q_{1}\right) i S_{F}\left(q_{1}-q\right) \Gamma\right], \\
& \tau^{a} \Gamma \rightarrow \tau^{a} \Gamma+i \gamma_{5} \tau^{a} \frac{2 i g}{1-2 g \Pi_{P S}\left(q^{2}\right)} \\
& \times\left(-2 N_{c}\right) \int \frac{d^{4} q_{1}}{(2 \pi)^{4}} \operatorname{tr}\left[i S_{F}\left(q_{1}\right) i \gamma_{5} i S_{F}\left(q_{1}-q\right) \Gamma\right]
\end{aligned}
$$

where $q^{\mu}=p_{1}^{\mu}-p_{2}^{\mu}=(0, \mathbf{q})$.

In the vector current case, only the isoscalar current can be dressed in principle. However, it is also easily proved that such a contribution vanishes, as

$\int \frac{d^{4} q_{1}}{(2 \pi)^{4}} \operatorname{tr}\left[i S_{F}\left(q_{1}\right) i S_{F}\left(q_{1}-q\right) \gamma^{0}\right]=0$

Therefore, in the particular case of the vector current under investigation here, there is no dressing of the vertex. 


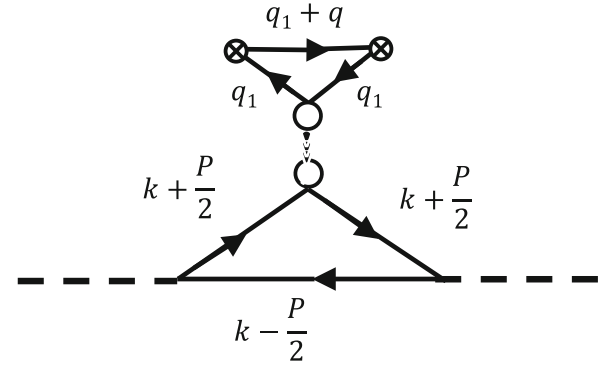

Fig. 3 Other type of contribution originated by the dressing of the currents

Finally, we consider the diagram depicted in Fig. 3. This contribution can be written in the form - $(p)$ stands for pole contribution,

$G_{\alpha \beta}^{(p) i j}(\mathbf{q})=\mathbb{M}_{i j} \frac{2 i g}{1-2 g \Pi_{S}(0)} \mathbb{N}_{\alpha \beta}$,

with

$$
\begin{aligned}
\mathbb{M}_{i j}= & -\int \frac{d^{4} \ell}{(2 \pi)^{4}} \operatorname{Tr}\left[\bar{\phi}_{\pi^{j}} i S_{F}(P+\ell) i S_{F}(P+\ell) \phi_{\pi^{i}} i S_{F}(\ell)\right] \\
& -\int \frac{d^{4} \ell}{(2 \pi)^{4}} \operatorname{Tr}\left[\bar{\phi}_{\pi^{j}} i S_{F}(\ell) \phi_{\pi^{i}} i S_{F}(\ell-P) i S_{F}(\ell-P)\right],
\end{aligned}
$$

and

$$
\begin{aligned}
\mathbb{N}_{\alpha \beta}= & -\int \frac{d^{4} k}{(2 \pi)^{4}} \operatorname{Tr}\left[i S_{F}(k) i S_{F}(k) \tau^{\beta} \Gamma i S_{F}(k+q) \tau^{\alpha} \Gamma\right] \\
& +\left(q^{\mu} \leftrightarrow-q^{\mu}, \alpha \leftrightarrow \beta\right) .
\end{aligned}
$$

The direct calculation gives, for $\mathbb{M}_{i j}$,

$\mathbb{M}_{i j}=\delta_{i j} 16 N_{c} g_{\pi q q}^{2} m\left[m_{\pi}^{2} I_{3}\left(m_{\pi}^{2}\right)-I_{2}(0)\right]$,

with $I_{2}\left(q^{2}\right)$ and $I_{3}\left(q^{2}\right)$ defined in Appendix A. The two quark intermediate state is described by the quantity,

$\frac{2 i g}{1-2 g \Pi_{S}(0)}=\frac{-i}{4 N_{c}\left[m_{\pi}^{2} I_{2}\left(m_{\pi}^{2}\right)+4 m^{2} I_{2}(0)\right]}$,

and for $\mathbb{N}_{\alpha \beta}$ we have

$\mathbb{N}_{\alpha \beta}=\delta_{\alpha \beta} i 2 N_{c}\left[I_{\Gamma}^{p}(m, \mathbf{q})+I_{\Gamma}^{p}(m,-\mathbf{q})\right]$,

with

$I_{\Gamma}^{p}(m, \mathbf{q})=\int \frac{d^{4} k}{(2 \pi)^{4}} \operatorname{tr}\left[S_{F}(k) S_{F}(k) \Gamma S_{F}(k+q) \Gamma\right]$.
Putting all these results together, we obtain

$$
\begin{aligned}
G_{\alpha \beta}^{(p) i j}(\mathbf{q})= & \delta_{i j} \delta_{\alpha \beta} 2 N_{c}\left(i g_{\pi q q}\right)^{2}\left(-4 m^{2}\right) \frac{\left[m_{\pi}^{2} I_{3}\left(m_{\pi}^{2}\right)-I_{2}(0)\right]}{\left[m_{\pi}^{2} I_{2}\left(m_{\pi}^{2}\right)+4 m^{2} I_{2}(0)\right]} \\
& \times\left[I_{\Gamma}^{p}(m, \mathbf{q})+I_{\Gamma}^{p}(m,-\mathbf{q})\right] .
\end{aligned}
$$

By comparing to the structures for the isospin amplitudes defined in Eq. (6), it is observed that the pole contributions add to the bare leading-order results in Eq. (20) in the following manner

$$
\begin{aligned}
& F_{0}(\mathbf{q})=X_{\Gamma}^{r}(\mathbf{q})+X_{\Gamma}^{t}(\mathbf{q})+X_{\Gamma}^{p}(\mathbf{q}), \\
& F_{1}(\mathbf{q})=-X_{\Gamma}^{r}(\mathbf{q})+X_{\Gamma}^{t}(\mathbf{q})+X_{\Gamma}^{p}(\mathbf{q}), \\
& F_{2}(\mathbf{q})=X_{\Gamma}^{r}(\mathbf{q}),
\end{aligned}
$$

with

$$
\begin{aligned}
X_{\Gamma}^{p}(\mathbf{q})= & 8 N_{c} g_{\pi q q}^{2} m^{2} \frac{\left[m_{\pi}^{2} I_{3}\left(m_{\pi}^{2}\right)-I_{2}(0)\right]}{\left[m_{\pi}^{2} I_{2}\left(m_{\pi}^{2}\right)+4 m^{2} I_{2}(0)\right]} \\
& \times\left[I_{\Gamma}^{p}(m, \mathbf{q})+I_{\Gamma}^{p}(m,-\mathbf{q})\right] .
\end{aligned}
$$

In the case $\Gamma=\gamma^{0}$, shown in the following as an example, $I_{\Gamma}^{p}(m, \mathbf{q})$ is a function of $q=|\mathbf{q}|$,

$I_{V^{0}}^{p}(m, q)=\int \frac{d k}{(2 \pi)^{2}} \frac{-k}{12 q E_{k}^{3}}\left(E_{k+q}-E_{k-q}\right)^{3}$,

and, after regularization, the last integral becomes

$\bar{I}_{V^{0}}^{p}(q)=\sum_{i=0}^{2} c_{i} I_{V^{0}}^{p}\left(M_{i}, q\right)$.

Putting all these results together we have finally

$$
\begin{aligned}
X_{V^{0}}^{p}(q)= & 16 N_{c} g_{\pi q q}^{2} m^{2} \\
& \times \frac{\left[m_{\pi}^{2} I_{3}\left(m_{\pi}^{2}\right)-I_{2}(0)\right]}{\left[m_{\pi}^{2} I_{2}\left(m_{\pi}^{2}\right)+4 m^{2} I_{2}(0)\right]} \bar{I}_{V^{0}}^{p}(q),
\end{aligned}
$$

and the final result for the functions under investigation, including all the contributions evaluated so far, can be summarized as follows

$$
\begin{aligned}
& F_{0}(q)=X_{V^{0}}^{r}(q)+X_{V^{0}}^{t}(q)+X_{V^{0}}^{p}(q), \\
& F_{1}(q)=-X_{V^{0}}^{r}(q)+X_{V^{0}}^{t}(q)+X_{V^{0}}^{p}(q), \\
& F_{2}(q)=X_{V^{0}}^{r}(q) .
\end{aligned}
$$




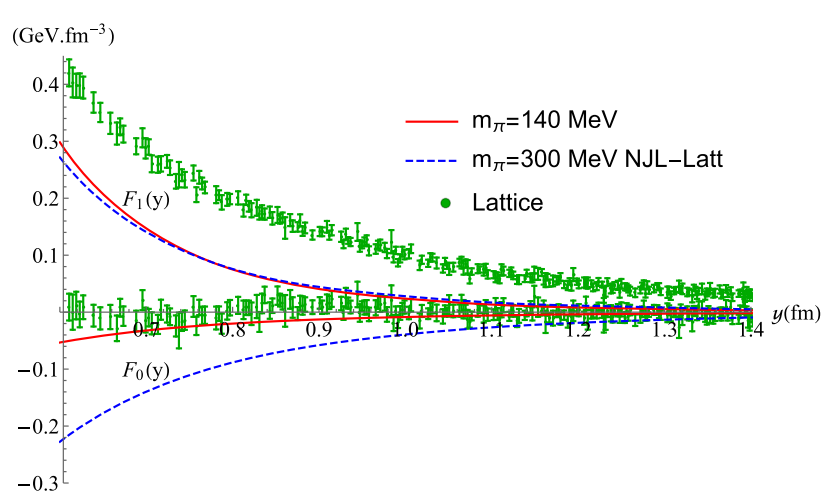

Fig. 4 The quantities $F_{0}$ and $F_{1}$, Eq. (43), in coordinate space, for various choices of the NJL parameters - given in Appendix A - corresponding to a different pion mass, to allow a proper comparison with lattice data [31] (green points with error band), for $\Gamma=\gamma^{0}$

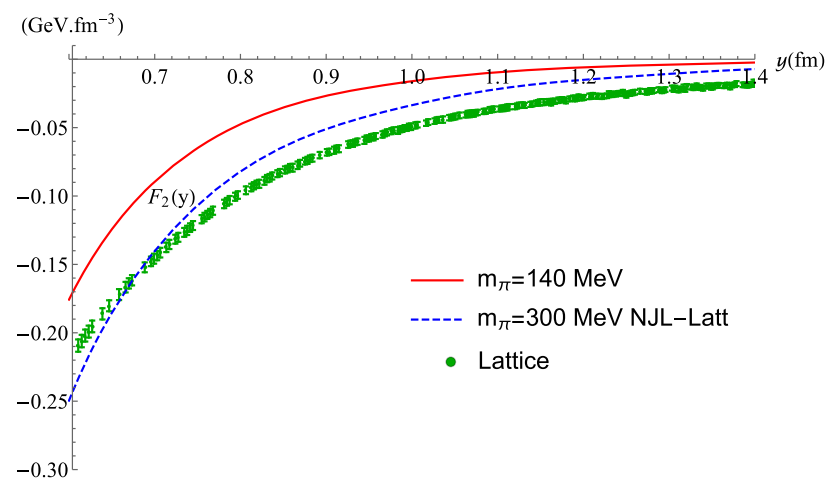

Fig. 5 Same as in Fig. 4, but for the function $F_{2}$ in Eq. (43)

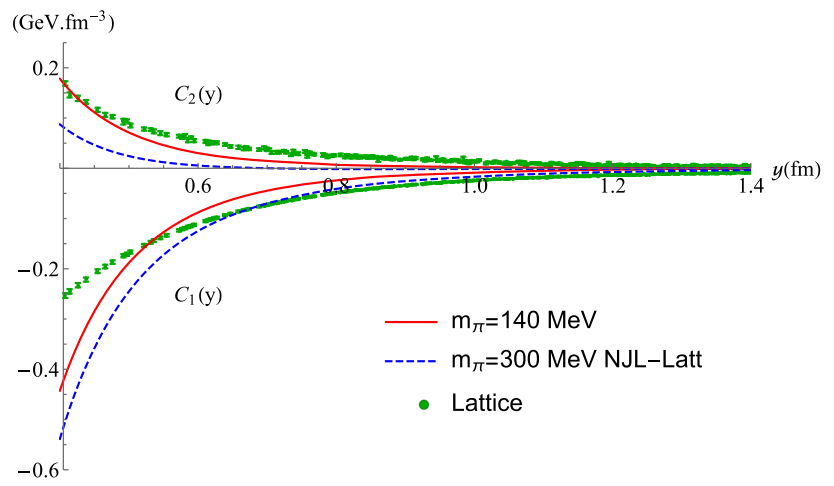

Fig. 6 The functions $C_{1}$ and $C_{2}$, Eq. (44), for various choices of the NJL parameters - given in Appendix A - corresponding to a different pion mass to allow a proper comparison with lattice data [31] (green points with error band), for $\Gamma=\gamma^{0}$, in coordinate space

\section{Comparison with lattice data}

The comparison of our results with the lattice evaluation of Ref. [31] in the vector-vector case is presented in Figs. 4, 5, 6 and 7.

The quantities $F_{0}$ and $F_{1}$ in Eq. (43) - evaluated using Eqs. (27) and (42) - are shown for $\Gamma=\gamma^{0}$ in Fig. 4,

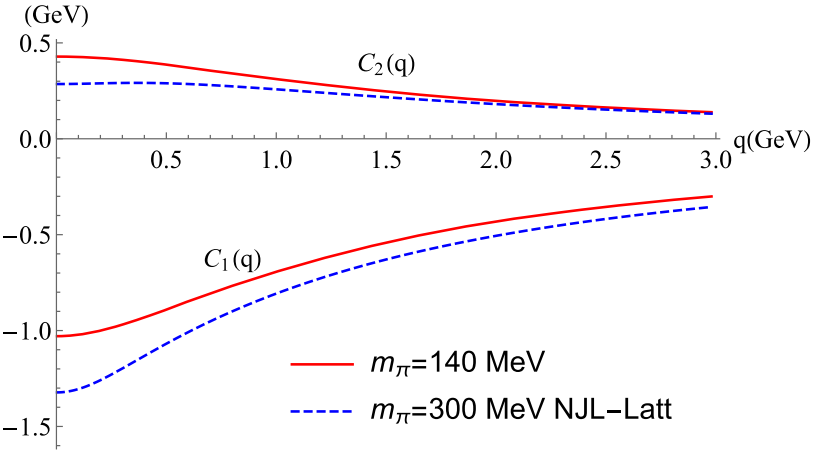

Fig. 7 Same as in Fig. 6, but in momentum space

in coordinate space, for various choices of the parameters of the NJL model corresponding to a different pion mass. The sets of model parameters corresponding to different values of the pion mass are reported in Appendix A. The change of the full parameter set allows a proper comparison with lattice data. The blue dashed curves - labelled $m_{\pi}=300 \mathrm{MeV} \mathrm{NJL}-L a t t$ - have to be compared with the lattice results - in green. Those lattice data correspond to Figs. 20 (c) and (a) of Ref. [31], respectively. A qualitative agreement is clearly found. A similar conclusion holds for $F_{2}$, given in Eq. (43) and shown in Fig. 5.

Besides, the results of the evaluations of the rhombus and trapezoid diagrams in our approach are related, in the calculation scheme of Ref. [31], to lattice contractions called $C_{1}$ and $C_{2}$. The correspondence formally reads

$$
\begin{aligned}
& C_{1}(q)=\frac{1}{2} X_{\Gamma}^{r}(q), \\
& C_{2}(q)=\frac{1}{4}\left[X_{\Gamma}^{t}(q)+X_{\Gamma}^{p}(q)\right] .
\end{aligned}
$$

The latter quantities can also be directly compared to the findings of Ref. [31], which will come of useful for the interpretation of contributions from diagrams. Our results for these functions are shown in Figs. 6 and 7, in coordinate and momentum space, respectively. In particular the former results show an encouraging agreement with the lattice data reported, in coordinate space, in Figs. 11 (a) of Ref. [31]. Overall, the NJL scheme seems to tentatively reproduce the trend of the available lattice results.

Following an argument discussed in Ref. [29], it is observed that, in the absence of correlations, two-current distributions could be computed from the single-current ones. This factorization hypothesis is comparable to an argument that has often been used more recently for dPDFs - see, e.g., Ref. [4]. This hypothesis is examined in Ref. [31]. In particular, for the isospin combination $\left\langle\pi^{+}(P)\left|\mathcal{O}_{n}^{u u}(y) \mathcal{O}_{n}^{d d}(0)\right| \pi^{+}(P)\right\rangle$, inserting a complete set of intermediate states between the two currents and keeping, among them, only the pion ground state, it can be shown 


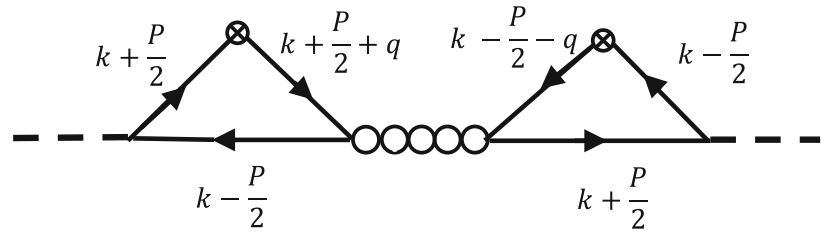

Fig. 8 One of the eight diagrams with pion intermediate states

that the two-current observable can be expressed in terms of single-current Form Factors.

In the most recent evaluation, the full lattice result for the $C_{1}$ contraction - as it dominates the relevant isospin combination - is compared to single-current expressions using known results for the charges - i.e. Form Factors at zero momentum transfer - as well as a common power law falloff for the momentum transfer dependence of the Form Factor. While the results at zero momentum transfer are acceptable, the attempt is found to generally fail to a large extent. Similarly an ansatz relates the dPDFs to the product of two GPDs, and we have analyzed it in the same model in Ref. [24]. Based on our previous experience, we expect a similar contribution for the two current correlation from diagrams of the type shown in Fig. 8, where the intermediate two quark state can have the quantum numbers of a $\sigma$ particle or a pion. Such contributions are allowed in the present scheme while not present in the lattice calculation. After a tedious evaluation detailed in the Appendix B for $\Gamma=\gamma^{0}$ and assuming dominance of the pion pole, in agreement with the procedure followed in Ref. [31], it is found that the only contribution arising from these diagrams can be written

$X_{\Gamma}^{F_{P S}^{-}}(\mathbf{q})=4 \frac{\left(E_{\pi}+m_{\pi}\right)^{2}}{E_{\pi}}\left[F_{e m}\left(2 m_{\pi}^{2}-2 m_{\pi} E_{\pi}\right)\right]^{2}$,

where $F_{e m}$ is the pion electromagnetic form factor and $E_{\pi}=\sqrt{m_{\pi}^{2}+\mathbf{q}^{2}}$. This expression - after using the relevant isospin combination from Eq. (B.6) - agrees with that adopted in Ref. [31] to approximate the full result given by the function $C_{1}$. As the contribution coming from diagram of Fig. 8 is not present in the lattice evaluation of $C_{1}$, in the NJL scheme used here we must discard it, in such a way that the only contribution to $C_{1}$ arise from the rhombus diagram. Therefore no comparison between the result of Eq. (45) with the contributions coming from $C_{1}$ - as suggested in Ref. [31] - can be made according to the spirit of the present paper. Accordingly we do not discuss relations based on that factorization hypothesis any further.

\section{Conclusions}

Two-partons correlations represent an elusive non perturbative information, theoretically encoded in two-current correlations as well as double parton distribution functions. The latter quantities are under theoretical investigation for the proton. There are perspectives to observe them during the LHC operation in the next years. While a possible access to the same observable for the pion appears challenging, lattice results have already been obtained for two-current correlations in the pion, a quantity that could be related to double parton distribution functions [31]. A consistent field-theoretical approach, based on the Nambu-Jona-Lasinio model with Pauli-Villars regularization, has been used in this paper for a systematic analysis of two-current correlations in the pion. We have given explicit expressions for the time-time component of the vector-vector two-current correlations in momentum space. We have compared our results for twocurrent correlations with lattice data, obtaining an overall good qualitative agreement. As happens in model calculation of the related quantities double parton distributions, it is found that, at the low scale relative to the model description, these functions encode novel non-perturbative information, not present in one-body quantities. This conclusion is highlighted in the consideration of a possible factorization of the two-current correlation into one-body Form Factors, which is found to be an unconnected contribution in our low-energy model. It will be interesting to compare our results for pion double distribution functions with forthcoming lattice data, whose evaluation is presently in progress [49].

Acknowledgements We thank M. Rinaldi for useful discussions and C. Zimmermann for sending us the lattice data of Ref. [31] relative to the quantities of interest here. This work was supported in part by the Mineco under contract FPA2016-77177-C2-1-P, by the STRONG-2020 project of the European Union Horizon 2020 research and innovation programme under grant agreement No 824093, and by UNAM through the PIIF project Perspectivas en Física de Partículas y Astropartículas as well as Grant No. DGAPA-PAPIIT IA101720. A.C. and S.S. thank the Department of Theoretical Physics of the University of Valencia for warm hospitality and support.

Data Availability Statement This manuscript has no associated data or the data will not be deposited. [Authors' comment: Lattice data are available in Ref. [31].]

Open Access This article is licensed under a Creative Commons Attribution 4.0 International License, which permits use, sharing, adaptation, distribution and reproduction in any medium or format, as long as you give appropriate credit to the original author(s) and the source, provide a link to the Creative Commons licence, and indicate if changes were made. The images or other third party material in this article are included in the article's Creative Commons licence, unless indicated otherwise in a credit line to the material. If material is not included in the article's Creative Commons licence and your intended use is not permitted by statutory regulation or exceeds the permitted use, you will need to obtain permission directly from the copy- 
right holder. To view a copy of this licence, visit http://creativecomm ons.org/licenses/by/4.0/.

Funded by SCOAP ${ }^{3}$.

\section{Appendix A: Basic integrals and Pauli-Villars regular- ization scheme}

In Sect. 2, we have used the Pauli-Villars regularization in order to render the occurring integrals finite. The way to proceed in this method is: (1) remove from the numerator all the powers of the integrated momentum, which will be replaced by external momenta, and the mass of the constituent quark, $m$; (2) for each resulting integral, which is of the form

$\widetilde{I}_{n}(\mu(m))=\int \frac{d^{4} k}{(2 \pi)^{4}} \frac{1}{\left[k^{2}-\mu(m)^{2}+i \epsilon\right]^{n}}$,

make the substitution

$\widetilde{I}_{n}^{r}(\mu(m))=\sum_{j=0}^{2} c_{j} \widetilde{I}_{n}\left(\mu\left(M_{j}\right)\right)$,

with $M_{j}^{2}=m^{2}+j \Lambda^{2}, c_{0}=c_{2}=1$ and $c_{1}=-2$.

Following this procedure, we obtain for the momentum integral of one propagator

$I_{1}=\frac{1}{16 \pi^{2}} \sum_{j=0}^{2} c_{j} M_{j}^{2} \ln \frac{M_{j}^{2}}{m^{2}}$,

and for the one of two propagators

$$
\begin{aligned}
I_{2}\left(m, q^{2}\right)= & \frac{1}{16 \pi^{2}} \sum_{j=0}^{2} c_{j} \\
& \times\left(\ln \frac{M_{j}^{2}}{m^{2}}+2 \sqrt{\frac{4 M_{j}^{2}}{q^{2}}-1} \arctan \frac{1}{\sqrt{\frac{4 M_{j}^{2}}{q^{2}}-1}}\right) .
\end{aligned}
$$

With the conventional values $\langle\bar{u} u\rangle=-(0.250 \mathrm{GeV})^{3}, f_{\pi}=$ $0.0924 \mathrm{GeV}$ and $m_{\pi}=0.140 \mathrm{GeV}$, we get $m=0.238 \mathrm{GeV}$, $\Lambda=0.860 \mathrm{GeV}$ and $m_{0}=5.4 \mathrm{MeV}$. For the pion-quark coupling constant we get $g_{\pi q q}^{2}=6.279$. We can obtain the chiral limit taking $m_{0}=0$, without changing $\Lambda$ and $m$. In that case $\langle\bar{u} u\rangle$ and $f_{\pi}$ do not change but one has $m_{\pi}=0$ and $g_{\pi q q}^{2}=6.625$.

For a proper comparison with the lattice data of Ref. [31], we have applied the same model with $\Lambda=1.022 \mathrm{GeV}$, $m=0.242 \mathrm{GeV}$ and $m_{0}=20 \mathrm{MeV}$, which lead to a massive pion with $m_{\pi}=0.300 \mathrm{GeV}, f_{\pi}=0.1 \mathrm{GeV}$ and $\langle\bar{u} u\rangle=-(0.285 \mathrm{GeV})^{3}$, as used in Ref. [31]. In this case we have $g_{\pi q q}^{2}=4.527$.

\section{Appendix B: Intermediate pion states}

Let us consider now the evaluation of the calculation of the process depicted in Fig. 8. There are eight different diagrams of this type.

This contribution can be written in the following form

$$
\begin{aligned}
& G_{\alpha \beta}^{(F) i j}(\mathbf{q}) \\
& =\int \frac{d q^{0}}{2 \pi}\left[\mathbb{U}_{j \beta} \frac{2 i g}{1-2 g \Pi_{S}\left((P+q)^{2}\right)} \mathbb{V}_{i \alpha}\right. \\
& \left.\quad+\left(q^{\mu} \leftrightarrow-q^{\mu}, \alpha \leftrightarrow \beta\right)\right] \\
& \quad+\int \frac{d q^{0}}{2 \pi}\left[\sum_{c=1}^{3} \widetilde{\mathbb{U}}_{j \beta c} \frac{2 i g}{1-2 g \Pi_{P S}\left((P+q)^{2}\right)} \widetilde{\mathbb{V}}_{i \alpha c}\right. \\
& \left.\quad+\left(q^{\mu} \leftrightarrow-q^{\mu}, \alpha \leftrightarrow \beta\right)\right] .
\end{aligned}
$$

The first (second) line of this equation describes an intermediate two quark state with the quantum numbers of a $\sigma(\pi)$ particle. In the case of the intermediate pion the additional isospin index, $c$, corresponds to its isospin. The quantities introduced in Eq. (B.1) are

$$
\begin{aligned}
\mathbb{U}_{j \beta}= & -\int \frac{d^{4} k}{(2 \pi)^{4}} \operatorname{Tr}\left[\bar{\phi}_{\pi^{j}} i S_{F}\right. \\
& \left.\times\left(k+\frac{P}{2}\right) \tau^{\beta} \Gamma i S_{F}\left(k+\frac{P}{2}+q\right) i S_{F}\left(k-\frac{P}{2}\right)\right] \\
& -\int \frac{d^{4} k}{(2 \pi)^{4}} \operatorname{Tr}\left[\bar{\phi}_{\pi^{j}} i S_{F}\left(k+\frac{P}{2}\right) i S_{F}\right. \\
& \left.\times\left(k-\frac{P}{2}-q\right) \tau^{\beta} \Gamma i S_{F}\left(k-\frac{P}{2}\right)\right] \\
\mathbb{V}_{i \alpha}= & -\int \frac{d^{4} k}{(2 \pi)^{4}} \operatorname{Tr}\left[\phi_{\pi^{i}} i S_{F}\right. \\
& \left.\times\left(k-\frac{P}{2}\right) i S_{F}\left(k+\frac{P}{2}+q\right) \tau^{\alpha} \Gamma i S_{F}\left(k+\frac{P}{2}\right)\right] \\
& -\int \frac{d^{4} k}{(2 \pi)^{4}} \operatorname{Tr}\left[\phi_{\pi^{i}} i S_{F}\left(k-\frac{P}{2}\right) \tau^{\alpha} \Gamma i S_{F}\right. \\
& \left.\times\left(k-\frac{P}{2}-q\right) i S_{F}\left(k+\frac{P}{2}\right)\right], \\
\widetilde{\mathbb{U}}_{j \beta c}= & -\int \frac{d^{4} k}{(2 \pi)^{4}} \operatorname{Tr}\left[\bar{\phi}_{\pi^{j}} i S_{F}\left(k+\frac{P}{2}\right) \tau^{\beta} \Gamma i S_{F}\right. \\
& \left.\times\left(k+\frac{P}{2}+q\right) i \gamma_{5} \tau^{c} i S_{F}\left(k-\frac{P}{2}\right)\right] \\
& -\int \frac{d^{4} k}{(2 \pi)^{4}} \operatorname{Tr}\left[\bar{\phi}_{\pi^{j}} i S_{F}\left(k+\frac{P}{2}\right) i \gamma_{5} \tau^{c} i S_{F}\right. \\
& \left.\times\left(k-\frac{P}{2}-q\right) \tau^{\beta} \Gamma i S_{F}\left(k-\frac{P}{2}\right)\right], \\
\widetilde{\mathbb{V}}_{i \alpha c}= & -\int \frac{d^{4} k}{(2 \pi)^{4}} \operatorname{Tr}\left[\phi_{\pi^{i}} i S_{F}\left(k-\frac{P}{2}\right) i \gamma_{5} \tau^{c} i S_{F}\right.
\end{aligned}
$$




$$
\begin{aligned}
& \left.\times\left(k+\frac{P}{2}+q\right) \tau^{\alpha} \Gamma i S_{F}\left(k+\frac{P}{2}\right)\right] \\
& -\int \frac{d^{4} k}{(2 \pi)^{4}} \operatorname{Tr}\left[\phi_{\pi^{i}} i S_{F}\left(k-\frac{P}{2}\right) \tau^{\alpha} \Gamma i S_{F}\right. \\
& \left.\times\left(k-\frac{P}{2}-q\right) i \gamma_{5} \tau^{c} i S_{F}\left(k+\frac{P}{2}\right)\right] .
\end{aligned}
$$

Performing the isospin traces, we obtain the following structures (we disregard the $G_{a \mathrm{~s}}^{(F) i j}$ and $G_{\mathrm{s} a}^{(F) i j}$ cases):

$$
\begin{aligned}
G_{\mathrm{ss}}^{(F) i j}(\mathbf{q})= & -\delta_{i j} X_{\Gamma}^{F_{P S}^{+}}(\mathbf{q}), \\
G_{a b}^{(F) i j}(\mathbf{q})= & -\delta_{i j} \delta_{a b} X_{\Gamma}^{F_{P S}^{-}}(\mathbf{q}) \\
& +\left(\delta_{j a} \delta_{i b}+\delta_{j b} \delta_{i a}\right) \frac{1}{2}\left[X_{\Gamma}^{F_{S}}(\mathbf{q})+X_{\Gamma}^{F_{P S}^{-}}(\mathbf{q})\right] \\
& +\left(\delta_{j a} \delta_{i b}-\delta_{j b} \delta_{i a}\right) \frac{1}{2}\left[Z_{\Gamma}^{F_{S}}(\mathbf{q})-Z_{\Gamma}^{F_{P S}^{-}}(\mathbf{q})\right],
\end{aligned}
$$

with

$$
\begin{aligned}
X_{\Gamma}^{F_{S}}(\mathbf{q})= & \left(i g_{\pi q q}\right)^{2} 4 N_{c}^{2} \int \frac{d q^{0}}{2 \pi} \mathbb{H}_{\Gamma}^{\mathrm{U}^{+}}(q) \\
& \times \frac{2 i g}{1-2 g \Pi_{S}\left((P+q)^{2}\right)} \mathbb{H}_{\Gamma}^{\mathrm{V}^{+}}(q)+(\mathbf{q} \leftrightarrow-\mathbf{q}), \\
X_{\Gamma}^{F_{P S}^{+}}(\mathbf{q})= & \left(i g_{\pi q q}\right)^{2} 4 N_{c}^{2} \int \frac{d q^{0}}{2 \pi} \mathbb{H}_{\Gamma}^{\tilde{\mathrm{U}}^{+}}(q) \\
& \times \frac{2 i g}{1-2 g \Pi_{P S}\left((P+q)^{2}\right)} \mathbb{H}_{\Gamma}^{\widetilde{\mathrm{V}}^{+}}(q)+(\mathbf{q} \leftrightarrow-\mathbf{q}), \\
X_{\Gamma}^{F_{P S}^{-}}(\mathbf{q})= & \left(i g_{\pi q q}\right)^{2} 4 N_{c}^{2} \int \frac{d q}{2 \pi} \mathbb{H}_{\Gamma}^{\mathrm{U}^{-}}(q) \\
& \times \frac{2 i g}{1-2 g \Pi_{P S}\left((P+q)^{2}\right)} \mathbb{H}_{\Gamma}^{\widetilde{\mathrm{V}}^{-}}(q)+(\mathbf{q} \leftrightarrow-\mathbf{q}), \\
Z_{\Gamma}^{F_{S}}(\mathbf{q})= & \left(i g_{\pi q q}\right)^{2} 4 N_{c}^{2} \int \frac{d q}{2 \pi} \mathbb{H}_{\Gamma}^{\mathrm{U}^{+}}(q) \\
& \times \frac{2 i g}{1-2 g \Pi_{S}\left((P+q)^{2}\right)} \mathbb{H}_{\Gamma}^{\mathrm{V}^{+}}(q)-(\mathbf{q} \leftrightarrow-\mathbf{q}), \\
Z_{\Gamma}^{F_{P S}^{-}}(\mathbf{q})= & \left(i g_{\pi q q}\right)^{2} 4 N_{c}^{2} \int \frac{d q}{2 \pi} \mathbb{H}_{\Gamma}^{\mathbb{U}^{-}}(q) \\
& \times \frac{2 i g}{1-2 g \Pi_{P S}\left((P+q)^{2}\right)} \mathbb{H}_{\Gamma}^{\widetilde{\mathrm{V}}^{-}}(q)-(\mathbf{q} \leftrightarrow-\mathbf{q}),
\end{aligned}
$$

where

$$
\begin{aligned}
\mathbb{H}_{\Gamma}^{\ell^{ \pm}}(q)= & \int \frac{d^{4} k}{(2 \pi)^{4}} \frac{1}{\left[\left(k+\frac{P}{2}\right)^{2}-m^{2}+i \epsilon\right]\left[\left(k-\frac{P}{2}\right)^{2}-m^{2}+i \epsilon\right]} \\
& \times\left(\frac{t_{\Gamma}^{\ell_{1}}(k)}{\left[\left(k+\frac{P}{2}+q\right)^{2}-m^{2}+i \epsilon\right]} \pm \frac{t_{\Gamma}^{\ell_{2}}(k)}{\left[\left(k-\frac{P}{2}-q\right)^{2}-m^{2}+i \epsilon\right]}\right),
\end{aligned}
$$

$$
\begin{aligned}
& t_{\Gamma}^{\mathrm{U}_{1}}=\operatorname{tr}\left[\gamma_{5}\left(k+\frac{P}{2}+m\right) \Gamma\left(k+\frac{P}{2}+\not q+m\right)\left(k-\frac{P}{2}+m\right)\right], \\
& t_{\Gamma}^{\mathrm{U}_{2}}=\operatorname{tr}\left[\gamma_{5}\left(k+\frac{P^{\prime}}{2}+m\right)\left(k-\frac{P^{\prime}}{2}-\not q+m\right) \Gamma\left(k-\frac{P^{Y}}{2}+m\right)\right] \text {, } \\
& t_{\Gamma}^{\mathrm{V}_{1}}=\operatorname{tr}\left[\gamma_{5}\left(k-\frac{P^{\prime}}{2}+m\right)\left(k+\frac{P^{Y}}{2}+\not d+m\right) \Gamma\left(k+\frac{P^{Y}}{2}+m\right)\right] \text {, } \\
& t_{\Gamma}^{\mathrm{V}_{2}}=\operatorname{tr}\left[\gamma_{5}\left(k-\frac{P^{\prime}}{2}+m\right) \Gamma\left(k-\frac{P^{\prime}}{2}-\not d+m\right)\left(k+\frac{P^{\prime}}{2}+m\right)\right] \text {, } \\
& t_{\Gamma}^{\widetilde{\mathrm{U}}_{1}}=\operatorname{tr}\left[\gamma_{5}\left(\not k+\frac{P}{2}+m\right) \Gamma\left(\not k+\frac{P}{2}+\not d+m\right) \gamma_{5}\left(\not k-\frac{P}{2}+m\right)\right], \\
& t_{\Gamma}^{\widetilde{\mathrm{U}}_{2}}=\operatorname{tr}\left[\gamma_{5}\left(\not k+\frac{\not P}{2}+m\right) \gamma_{5}\left(\not k-\frac{\not P}{2}-\not \phi+m\right) \Gamma\left(\not k-\frac{\not Y}{2}+m\right)\right] \text {, } \\
& t_{\Gamma}^{\widetilde{\mathrm{V}}_{1}}=\operatorname{tr}\left[\gamma_{5}\left(\not k-\frac{\not P}{2}+m\right) \gamma_{5}\left(\not k+\frac{\not P}{2}+\not q+m\right) \Gamma\left(\not k+\frac{\not P}{2}+m\right)\right] \text {, } \\
& t_{\Gamma}^{\widetilde{\mathrm{V}}_{2}}=\operatorname{tr}\left[\gamma_{5}\left(\not k-\frac{\not P}{2}+m\right) \Gamma\left(\not k-\frac{P}{2}-\not q+m\right) \gamma_{5}\left(\not k+\frac{\not P}{2}+m\right)\right] .
\end{aligned}
$$

The intermediate two quark states appearing in Eq. (B.7) can be approximated by the quantities -the relevant expressions are available, e.g. in Ref. [24]

$$
\begin{aligned}
\frac{2 i g}{1-2 g \Pi_{S}\left((P+q)^{2}\right)} & \simeq \frac{-i g_{\sigma q q}^{2}}{(P+q)^{2}-m_{\sigma}^{2}+i \epsilon}, \\
\frac{2 i g}{1-2 g \Pi_{P S}\left((P+q)^{2}\right)} & \simeq \frac{-i g_{\pi q q}^{2}}{(P+q)^{2}-m_{\pi}^{2}+i \epsilon} .
\end{aligned}
$$

Let us consider, as always in this paper, the $\Gamma=\gamma^{0}$ case. Performing the traces present in Eq. (B.8) we have

$\mathbb{H}_{V^{0}}^{\mathrm{U}^{+}}(q)=\mathbb{H}_{V^{0}}^{\mathrm{V}^{+}}(q)=\mathbb{H}_{V^{0}}^{\widetilde{\mathrm{U}}^{+}}(q)=\mathbb{H}_{V^{0}}^{\widetilde{\mathrm{V}}^{+}}(q)=0$,

so that $X_{V^{0}}^{F_{S}}(\mathbf{q})=X_{V^{0}}^{F_{P S}^{+}}(\mathbf{q})=Z_{\Gamma}^{F_{S}}(\mathbf{q})=0$. After a tedious but straightforward calculation we obtain,

$$
\begin{aligned}
\mathbb{H}_{\Gamma}^{\widetilde{\mathrm{U}}^{-}}(q) & =\mathbb{H}_{\Gamma}^{\widetilde{\mathrm{V}}^{-}}(q) \\
& =\frac{1}{N_{c} g_{\pi q q}^{2}}\left[(2 P+q)^{0} F_{e m}\left(q^{2}\right)+q^{0} \widetilde{F}_{e m}\left(q^{2}\right)\right],
\end{aligned}
$$

where $F_{e m}\left(q^{2}\right)$ and $\widetilde{F}_{e m}\left(q^{2}\right)$ are

$$
\begin{aligned}
F_{e m}\left(q^{2}\right)= & \frac{2 N_{c} g_{\pi q q}^{2}}{P^{2} P^{\prime 2}-\left(P \cdot P^{\prime}\right)^{2}}\left[P^{2}\left(P \cdot P^{\prime}-P^{\prime 2}\right) I_{2}\left(P^{2}\right)\right. \\
& +P^{\prime 2}\left(P \cdot P^{\prime}-P^{2}\right) I_{2}\left(P^{\prime 2}\right)+\left(P \cdot P^{\prime}\right) \\
& \left(2 P \cdot P^{\prime}-P^{2}-P^{\prime 2}\right) I_{2}\left(\left(P-P^{\prime}\right)^{2}\right)-P^{2} P^{\prime 2} \\
& \left.\left(2 P \cdot P^{\prime}-P^{2}-P^{\prime 2}\right) I_{3}\left(P, P^{\prime}\right)\right], \quad(\mathrm{B} .14) \\
\widetilde{F}_{e m}\left(q^{2}\right)= & \frac{2 N_{c} g_{\pi q q}^{2}}{P^{2} P^{\prime 2}-\left(P \cdot P^{\prime}\right)^{2}}\left[P^{2}\left(P \cdot P^{\prime}-P^{\prime 2}\right) I_{2}\left(P^{2}\right)\right. \\
& -P^{\prime 2}\left(P \cdot P^{\prime}-P^{2}\right) I_{2}\left(P^{\prime 2}\right)
\end{aligned}
$$




$$
\begin{aligned}
& +\left(P \cdot P^{\prime}\right)\left(P^{\prime 2}-P^{2}\right) I_{2}\left(\left(P-P^{\prime}\right)^{2}\right) \\
& \left.-P^{2} P^{\prime 2}\left(P^{\prime 2}-P^{2}\right) I_{3}\left(P, P^{\prime}\right)\right],
\end{aligned}
$$

with $P^{\prime \mu}=P^{\mu}+q^{\mu}$. These two form factors correspond to the standard electromagnetic form factors,

$$
\begin{aligned}
& \left\langle\pi(P)\left|J_{e m}^{\mu}\right| \pi\left(P^{\prime}\right)\right\rangle \\
& \quad=\left(P^{\prime}+P\right)^{\mu} F_{e m}\left(q^{2}\right)+\left(P^{\prime}-P\right)^{\mu} \widetilde{F}_{e m}\left(q^{2}\right) .
\end{aligned}
$$

When both pions are on-shell, $\widetilde{F}_{e m}\left(q^{2}\right)$ vanishes and $F_{\text {em }}\left(q^{2}\right)$ reads

$$
\begin{aligned}
F_{e m}\left(q^{2}\right)= & \frac{4 N_{c} g_{\pi q q}^{2}}{m_{\pi}^{2}+\left(P \cdot P^{\prime}\right)}\left[-m_{\pi}^{2} I_{2}\left(m_{\pi}^{2}\right)\right. \\
& \left.-\left(P \cdot P^{\prime}\right) I_{2}\left(\left(P-P^{\prime}\right)^{2}\right)+m_{\pi}^{4} I_{3}\left(P, P^{\prime}\right)\right]
\end{aligned}
$$

Putting together all these ingredients we have

$$
\begin{aligned}
X_{\Gamma}^{F_{P S}^{-}}(\mathbf{q})= & 8 i \int \frac{d q^{0}}{2 \pi} \\
& \times \frac{\left[(2 P+q)^{0} F_{e m}\left(q^{2}\right)+q^{0} \widetilde{F}_{e m}\left(q^{2}\right)\right]^{2}}{(P+q)^{2}-m_{\pi}^{2}+i \epsilon},
\end{aligned}
$$

$Z_{\Gamma}^{F_{P S}^{-}}(\mathbf{q})=0$.

The $q^{0}$ integral will be dominated by the pole contribution and we obtain the final result

$X_{\Gamma}^{F_{P S}^{-}}(\mathbf{q})=4 \frac{\left(E_{\pi}+m_{\pi}\right)^{2}}{E_{\pi}}\left[F_{e m}\left(2 m_{\pi}^{2}-2 m_{\pi} E_{\pi}\right)\right]^{2}$,

which is the expression reported at the end of Sect. 4 .

\section{References}

1. P. Bartalini, J.R. Gaunt, Adv. Ser. Direct. High Energy Phys. 29, 1 (2018). https://doi.org/10.1142/10646

2. G. Aad et al., ATLAS Collaboration, New J. Phys. 15, 033038 (2013)

3. N. Paver, D. Treleani, Nuovo Cim. A 70, 215 (1982)

4. M. Diehl, D. Ostermeier, A. Schafer, JHEP 03, 089 (2012)

5. M. Guidal, H. Moutarde, M. Vanderhaeghen, Rep. Prog. Phys. 76, 066202 (2013)

6. R. Dupré, M. Guidal, M. Vanderhaeghen, Phys. Rev. D 95(1), 011501 (2017)

7. C.A. Aidala et al. arXiv:2002.12333 [hep-ph]

8. B. Blok, Y. Dokshitser, L. Frankfurt, M. Strikman, Eur. Phys. J. C 72, 1963 (2012)

9. B. Blok, Y. Dokshitzer, L. Frankfurt, M. Strikman, Eur. Phys. J. C 74, 2926 (2014)

10. A. Del Fabbro, D. Treleani, Phys. Rev. D 63, 057901 (2001)

11. M. Rinaldi, F.A. Ceccopieri, Phys. Rev. D 97(7), 071501 (2018)

12. M. Rinaldi, F.A. Ceccopieri, JHEP 1909, 097 (2019)

13. T. Kasemets, S. Scopetta, Adv. Ser. Direct. High Energy Phys. 29, 49 (2019)
14. H.M. Chang, A.V. Manohar, W.J. Waalewijn, Phys. Rev. D 87(3), 034009 (2013)

15. M. Rinaldi, S. Scopetta, V. Vento, Phys. Rev. D 87, 114021 (2013)

16. M. Rinaldi, S. Scopetta, M. Traini, V. Vento, JHEP 12, 028 (2014)

17. T. Kasemets, A. Mukherjee, Phys. Rev. D 94(7), 074029 (2016)

18. M. Rinaldi, S. Scopetta, M. Traini, V. Vento, Phys. Lett. B 752, 40 (2016)

19. M. Rinaldi, S. Scopetta, M.C. Traini, V. Vento, JHEP 16, 063 (2016)

20. M. Traini, M. Rinaldi, S. Scopetta, V. Vento, Phys. Lett. B 768, 270 (2017)

21. M. Diehl, J.R. Gaunt, Adv. Ser. Direct. High Energy Phys. 29, 7 (2018)

22. M. Rinaldi, S. Scopetta, M. Traini, V. Vento, Eur. Phys. J. C 78(9), 781 (2018)

23. M. Rinaldi, Eur. Phys. J. C 80(7), 678 (2020). https://doi.org/10. 1140/epjc/s10052-020-8241-y. arXiv:2003.09400 [hep-ph]

24. A. Courtoy, S. Noguera, S. Scopetta, JHEP 1912, 045 (2019)

25. W. Broniowski, E. Ruiz Arriola, Phys. Rev. D 101(1), 014019 (2020)

26. H.W. Lin et al., Prog. Part. Nucl. Phys. 100, 107-160 (2018). https://doi.org/10.1016/j.ppnp.2018.01.007. arXiv:1711.07916 [hep-ph]

27. X. Ji, Phys. Rev. Lett. 110, 262002 (2013). https://doi.org/10.1103/ PhysRevLett.110.262002. arXiv:1305.1539 [hep-ph]

28. H.W. Lin et al., arXiv:2006.08636 [hep-ph]

29. M. Burkardt, J. Grandy, J.W. Negele, Ann. Phys. 238, 441-472 (1995). https://doi.org/10.1006/aphy.1995.1026. arXiv:hep-lat/9406009 [hep-lat]

30. C. Zimmermann, RQCD Collaboration, PoS LATTICE 2016, 152 (2016)

31. G.S. Bali et al., JHEP 1812, 061 (2018)

32. R.M. Davidson, E. Ruiz Arriola, Acta Phys. Polon. B 33, 1791 (2002)

33. L. Theussl, S. Noguera, V. Vento, Eur. Phys. J. A 20, 483 (2004)

34. E. Ruiz Arriola, W. Broniowski, Phys. Rev. D 66, 094016 (2002)

35. A. Courtoy, S. Noguera, Phys. Lett. B 675, 38 (2009)

36. A. Courtoy, S. Noguera, Phys. Rev. D 76, 094026 (2007)

37. A. Courtoy, Ph.D. Thesis, Valencia University (2009). arXiv: 1010.2974 [hep-ph]

38. S. Noguera, S. Scopetta, Phys. Rev. D 85, 054004 (2012)

39. H. Weigel, E. Ruiz Arriola, L.P. Gamberg, Nucl. Phys. B 560, 383 (1999)

40. S. Noguera, S. Scopetta, JHEP 1511, 102 (2015)

41. W. Broniowski, E. Ruiz Arriola, Phys. Rev. D 97(3), 034031 (2018)

42. F.A. Ceccopieri, A. Courtoy, S. Noguera, S. Scopetta, Eur. Phys. J. C 78(8), 644 (2018)

43. S.P. Klevansky, Rev. Mod. Phys. 64, 649 (1992)

44. V. Bernard, U.G. Meissner, A.A. Osipov, Phys. Lett. B 324, 201-208 (1994). https://doi.org/10.1016/0370-2693(94)90408-1. arXiv:hep-ph/9312203 [hep-ph]

45. U. Vogl, M.F.M. Lutz, S. Klimt, W. Weise, Nucl. Phys. A 516, 469-495 (1990). https://doi.org/10.1016/0375-9474(90)90124-5

46. J. Bijnens, Phys. Rep. 265, 369-446 (1996). https://doi.org/10. 1016/0370-1573(95)00051-8. arXiv:hep-ph/9502335 [hep-ph]

47. M. Stratmann, Z. Phys. C 60, 763-772 (1993). https://doi.org/10. 1007/BF01558408

48. M. Traini, A. Mair, A. Zambarda, V. Vento, Nucl. Phys. A 614, 472 500 (1997). https://doi.org/10.1016/S0375-9474(96)00450-2

49. C. Zimmermann, private communication 